\title{
Technique and diagnostic utility of saline infusion sonohysterography
}

\author{
Khaled M. Elsayes *, Amit Pandya, Joel F. Platt, Ronald O. Bude \\ Department of Radiology, University of Michigan Health Center, Ann Arbor, Michigan, USA
}

\section{A R T I C L E I N F O}

\section{Article history:}

Received 6 June 2008

Received in revised form 7 November 2008

Accepted 17 November 2008

\section{Keywords:}

Endometrium

Diagnostic utility

Saline infusion sonohysterography

Technique

\begin{abstract}
A B S T R A C T
The introduction of saline infusion sonohysterography has significantly improved sonographic diagnosis of various endometrial pathologies. This procedure entails instillation of warm saline into the uterine cavity transcervically to provide enhanced visualization of the endometrium during transvaginal ultrasound examination. This article reviews the sonohysterography technique and current utility, as well as the spectrum of imaging features of various endometrial pathologies.
\end{abstract}

(c) 2009 International Federation of Gynecology and Obstetrics. Published by Elsevier Ireland Ltd. All rights reserved.

\section{Introduction}

Saline infusion sonohysterography is a procedure in which warm saline is instilled into the uterine cavity to provide enhanced endometrial visualization during transvaginal ultrasound $[1,2]$. This technique improves detection of endometrial pathology, such as polyps, leiomyomas, hyperplasia, cancer, and adhesions. In addition, it helps avoid invasive diagnostic procedures as well as optimize the preoperative triage process for women requiring therapeutic intervention. It is easily and rapidly performed at reasonable cost, well tolerated, and virtually devoid of complications.

Sonohysterography is particularly useful for finding focal endometrial abnormalities or further confirming and defining the pathologic nature of a potential abnormality detected by ultrasound [3].

Sonohysterography is typically indicated in premenopausal women with abnormal vaginal bleeding because it can distinguish anovulatory bleeding, which is best treated hormonally, from an anatomic lesion, which may require tissue sampling and/or resection for treatment [4]. Sonohysterography is also indicated in postmenopausal women with abnormal vaginal bleeding [5], as it can distinguish bleeding due to atrophy (the most common cause of bleeding in this age group), from anatomic lesions that might require tissue sampling and/or resection for treatment.

Saline infusion sonohysterography is also useful for detecting potential anatomic causes of reduced fertility, such as submucosal myomas, endometrial polyps, anomalies, and intrauterine adhesions

\footnotetext{
* Corresponding author. Department of Radiology, University of Michigan Health Center at Ann Arbor, MI 48109-0030, USA. Tel: +1 734936 4489; fax: +1 7346470013. E-mail address: kelsayes@med.umich.edu (K.M. Elsayes).
}

[1,2,6-9]. The spectrum of indications and contraindications of sonohysterography is summarized in Table 1.

\section{Technique}

Sonohysterography is typically scheduled early in the follicular phase of the menstrual cycle, after cessation of menstrual flow and before day 10 , as the endometrium is thin at this point in the cycle. Later in the cycle, focal contour irregularities of the endometrium may be mistaken for small polyps or focal areas of endometrial hyperplasia.

Anesthesia or analgesia is not required for insertion of the intrauterine catheter since this is often painless. Although the procedure is usually tolerated by the majority of patients, a minority may experience some cramping, for which a nonsteroidal anti-inflammatory drug (NSAID) such as ibuprofen ( $400 \mathrm{mg}$ ) can be prescribed 30 minutes before the examination. For patients allergic to ibuprofen, ketorolac can be prescribed.

After confirming that the patient is not pregnant, procedural steps must be explained to ensure the patient's comfort, and consent must be obtained before proceeding. The patient should be placed in the lithotomy position. Sterile technique is preferable to prevent endometritis and other infections. A speculum is inserted into the vaginal introitus. The cervical os is localized and cleaned with a povidoneiodine solution or chlorhexidine gluconate.

A 5 or 7 French sonohysterography catheter should be flushed with sterile saline prior to insertion to clear it of air, which can cause an echogenic artifact in the uterine cavity. The catheter is then inserted through the cervical os into the cervical canal. Its balloon tip is inflated with 1-2 $\mathrm{mL}$ of saline, depending on patient comfort, to help hold it in place. The speculum is then removed carefully to avoid dislodging the catheter. Because the catheter is $25 \mathrm{~cm}$ long, it will still extend beyond the introitus when positioned in the uterus. Occasionally, it is difficult 
Table 1

Common indications and contraindications for sonohysterography

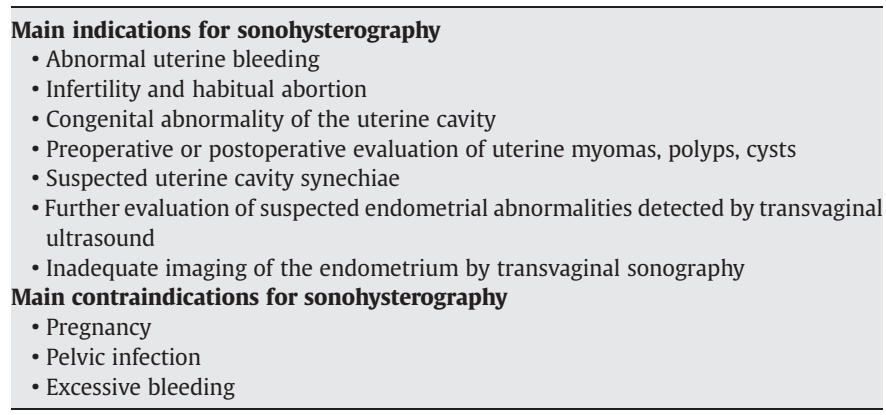

to thread the flexible catheter into place because of cervical stenosis, variable uterine position, or abnormal uterine contour. Changing the toe of the speculum by moving the handle up or down may change the angle of the cervix with the fundus and will often allow successful catheter insertion. If the cervical os can be seen but the catheter cannot be placed into it, two maneuvers may help. A cervical dilator may be used to dilate the cervical canal. If this fails to allow passage of the catheter it may be possible to pass a guidewire through the os and then pass a catheter without a balloon tip over the guidewire into the os. Lack of a balloon tip may not hinder adequate distension of the uterine cavity as the cervical stenosis may assist in retention of fluid within the canal. Careful attention should be given to the location and amount of balloon inflation because suboptimal placement and balloon overinflation can obscure visualization of an endometrial lesion (Fig. 1).

If the catheter cannot pass the cervical os it may be possible to obtain adequate images by placing the tip in the os and applying the filled balloon to the cervix.

The vaginal probe is then reinserted and a $10 \mathrm{~mL}$ syringe filled with sterile saline is attached to the catheter. Fluid is instilled while the transducer is moved from side to side (cornua to cornua) in a long-axis projection. The amount of instilled fluid varies, depending upon distension of the endometrial cavity as seen on the ultrasound monitor and also on patient comfort (cramps may occur). After the uterus is completely surveyed from cornua to cornua in a long-axis projection, the transducer is rotated 90 degrees into an axial plane. More fluid is instilled while fanning down toward the endocervical canal and up toward the uterine fundus to obtain a detailed survey of the endometrium. Each portion of the uterine cavity should be imaged, including the cervical canal in a coronal sweep, to exclude any focal abnormality, such as polyps, myoma, hyperplasia, and carcinoma.

Visualization of the endocervical canal is problematic because it is often difficult to distend this portion of the canal. Adequate visualization often requires a delicate progressive collapse of the balloon during fluid instillation into the canal and while the catheter is pulled back or passively slips out of the uterus (Fig. 2). This maneuver should be performed routinely, although it can be difficult to perform and is learned with experience. Transabdominal images during saline solution instillation sometimes provide an acceptable image. A reliable assessment requires that the endometrial echo is homogeneous and surrounded by an intact hypoechoic junctional zone.

\section{Complications}

Procedure-related adverse effects and complications are mild and uncommon. A prospective study of 1153 women undergoing saline infusion sonohysterography reported failure to complete the procedure (7\%), pelvic pain (3.8\%), vagal symptoms (3.5\%), nausea (1\%), and post procedure fever $(0.8 \%)$ [10]. Causes of incomplete procedures were the presence of a stenotic cervix which did not allow insertion of the intrauterine catheter, an insufficient cervical seal causing vaginal backflow of saline, and difficulty in distending the uterine cavity because of myomas [10].

\section{Sonohysterographic features of various endometrial pathologies}

It must be understood that the chief uses of sonohysterography are to determine if the uterine cavity is normal or abnormal and, if it is abnormal, whether the abnormality is focal or diffuse. Although there are "typical" features of various types of endometrial pathology, sonohysterography is often not adequate to determine pathology to accurately obviate biopsy. Polyps can look like fibroids and vice versa. Foci of endometrial carcinoma can be present in a lesion that looks like a polyp or endometrial hyperplasia. Therefore, the main value of this examination is to help the referring physician plan further work-up. If the endometrium appears "normal", no further work-up may be needed. If the endometrium is abnormal, the main utility of sonohysterography may be to direct the next type of intervention to be performed. If the abnormality is focal, direct hysteroscopic visualization and guided biopsy may be needed to ensure adequate

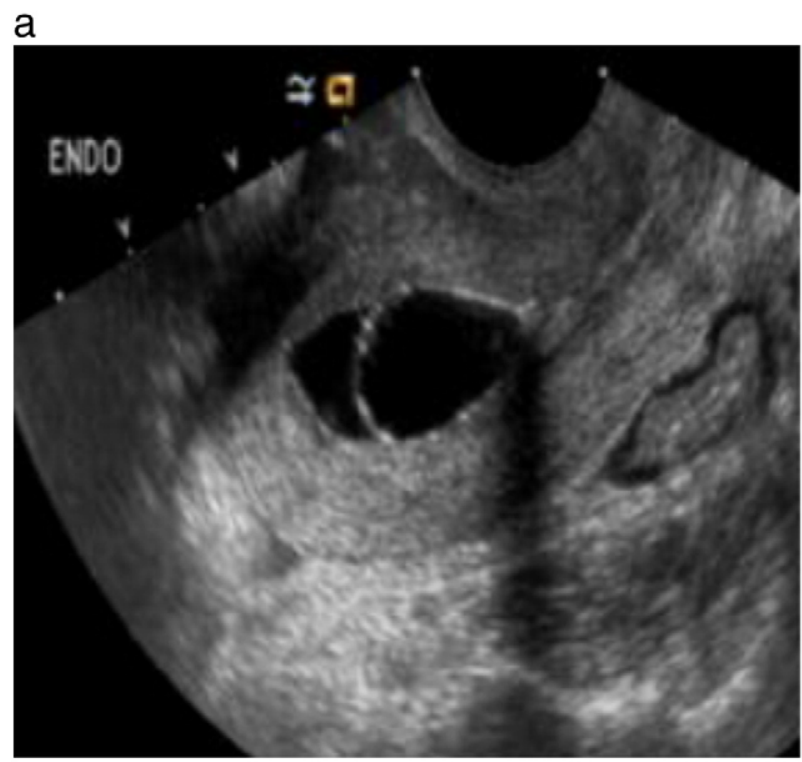

b

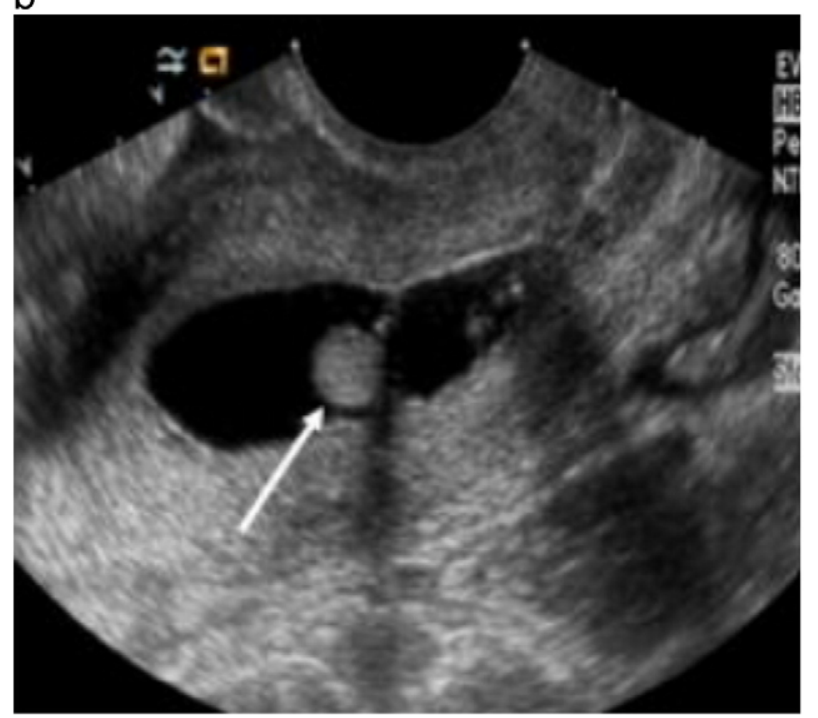

Fig. 1. 35-year-old female with a 6-month history of postcoital bleeding. Sonohysterogram shows importance of balloon placement. (a) Balloon was placed high within the endometrial cavity, obscuring visualization of an endometrial polyp which is well visualized after withdrawing and deflating the balloon (b). 


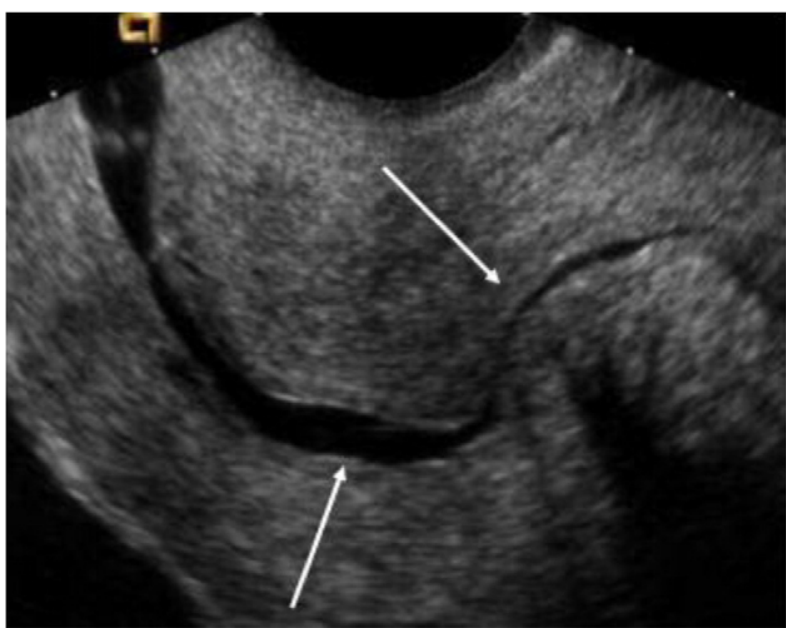

Fig. 2. Sonohysterogram adequately visualized a normal cervical canal after a delicate progressive collapse of the balloon during fluid instillation into the canal and while the catheter is pulled back and passively slips out of the uterus.

tissue sampling. If the abnormality is diffuse, dilatation and curettage may be sufficient for adequate tissue sample. Keeping these considerations in mind, fairly characteristic appearances of endometrial abnormalities are presented.

Endometrial pathologies can be subclassified according to their degree of involvement into focal and diffuse lesions. Focal lesions are defined as lesions occupying less than $25 \%$ of the endometrial surface area. On the other hand, diffuse lesions involve a greater percentage of the endometrial surface area.

\subsection{Endometrial polyp}

An endometrial polyp is a localized overgrowth of endometrial glands and stroma that project beyond the surface of the endometrium, with an incidence of $10 \%$ among women and a peak occurrence between 40 and 49 years of age. An endometrial polyp is soft, pliable, and may be single or multiple. Most polyps arise from the fundus of the uterus. Endometrial polyps vary from a few millimeters to several centimeters in diameter, and it is possible for a single large polyp to fill the entire endometrial cavity. Endometrial polyps may have a broad base (sessile) or can be attached by a slender pedicle (pedunculated) (Fig. 3a).

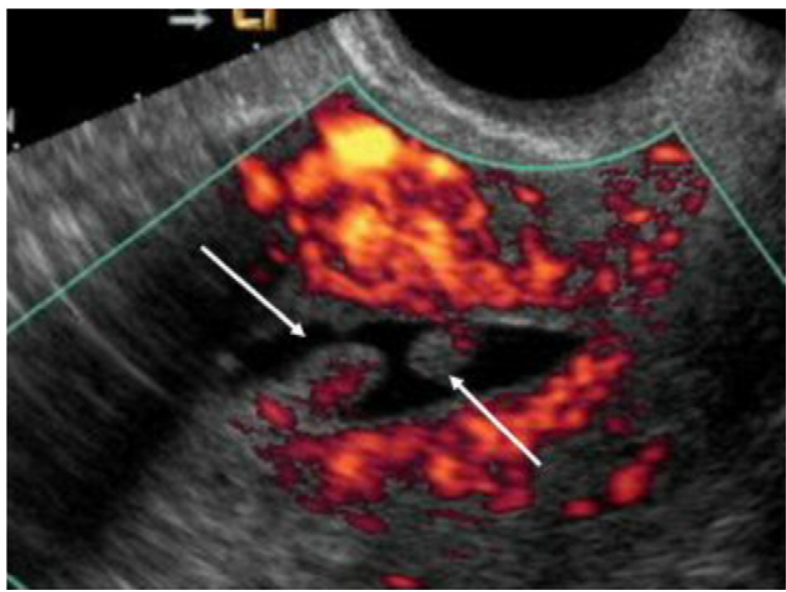

Fig. 3. 45-year-old female with abnormal vaginal bleeding. Sonohysterogram with color Doppler interrogation revealed 2 endometrial polyps projecting into the uterine cavity, 1 from the anterior wall with a narrow stalk and 1 from the posterior wall with a broader stalk. A central vessel is seen feeding each of these polyps (arrows).
The typical sonohysterographic appearance of an endometrial polyp is a well defined, homogeneous, rounded lesion isoechoic to the endometrium with preservation of the endometrial-myometrial interface [11]. Atypical features of an endometrial polyp include cystic components, multiple polyps, broad base, and hypoechogenicity or heterogeneity. This complex appearance may indicate hemorrhage, infarction, or inflammation within the polyp. Color flow or power Doppler can identify the common finding of a central feeder vessel (Fig. 3b). However, this is a nonspecific finding, because atypical fibroids or even endometrial carcinoma can also have this appearance.

\subsection{Leiomyoma}

Submucosal leiomyomas or fibroids are a common cause of abnormal uterine bleeding and are associated with reproductive dysfunction including recurrent miscarriage, infertility, premature labor, and fetal malpresentations, as well as obstructed labor [11]. Submucosal leiomyomas are also associated with postmenopausal bleeding, accounting for approximately $10 \%$ of such cases [1,2]. Sonohysterography usually depicts leiomyomas and accurately assesses their location, size, and degree of intramural extension. Submucosal leiomyomas are usually seen as well defined, broadbased, hypoechoic, solid masses which distort the endometrialmyometrial interface, with refractile shadowing often arising from within the mass (Fig. 4) [12]. The leiomyoma is usually covered with an overlying echogenic endometrial layer. Sonohysterography has an added advantage of better estimation of the percentage circumference projecting into the endometrial cavity [13]. This is important because if removal is planned, a greater than $50 \%$ projection of the leiomyoma into the uterine cavity suggests that hysteroscopic removal, instead of open removal, can be performed. Atypical appearances of leiomyomas seen at sonohysterography include pedunculation, and multilobulation, as well as prolapse into the endocervical canal which often precludes sonohysterography $[14,15]$. These appearances can be mistaken to indicate polyps if histologic analysis is not performed.

\subsection{Endometrial hyperplasia}

Endometrial hyperplasia is a pathologic term used to describe a group of proliferative disorders of the endometrium usually resulting from unopposed estrogenic stimulation, and accounting for approximately $4 \%-8 \%$ of postmenopausal bleeding cases [1].

Endometrial hyperplasia is histologically defined as an irregular proliferation of endometrial glands, with an increase in the glandstroma ratio compared with the normal proliferative endometrium

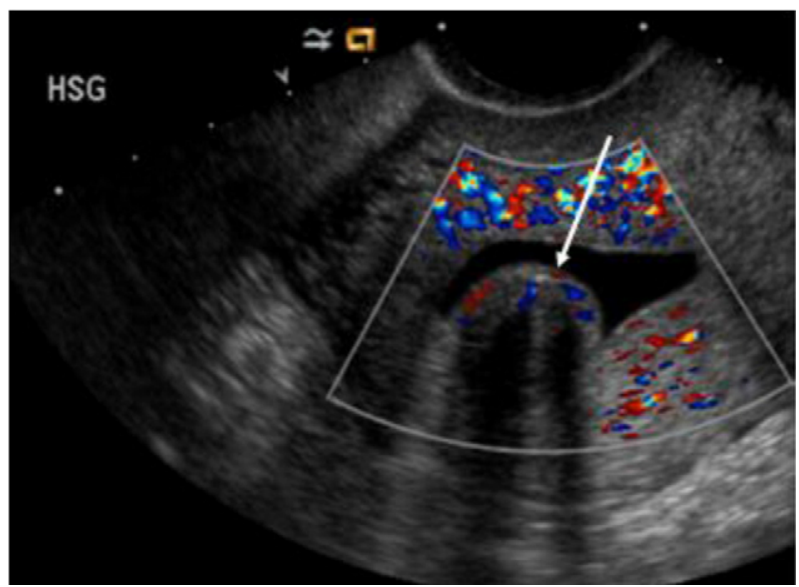

Fig. 4. 47-year-old female with dysfunctional uterine bleeding. Sonohysterogram with color Doppler interrogation showed a submucosal fibroid, manifested by broad-based heterogenous soft tissue projection in the uterine cavity with visualization of a thin echogenic endometrial lining (arrow). Multiple feeding vessels are noted. 
$[1,8]$. Risk factors for developing endometrial hyperplasias are similar to those for carcinoma, including exposure to unopposed estrogen (either endogenous or exogenous), tamoxifen usage, nulliparity, obesity, hypertension, and diabetes. There are various histologic patterns of endometrial hyperplasia, ranging from a hyperplasia without atypia, which has little or no malignant potential (less than $2 \%$ progress to carcinoma), to a severe atypia in which $20 \%$ of cases progress to endometrial cancer [1]. The greater the complexity of architecture, the higher the likelihood of cancer development. Endometrial hyperplasia usually appears as diffuse irregular echogenic endometrial thickening without focal abnormality, but the sonohysterographic appearance is not pathognomonic and biopsy is often required to establish the diagnosis and to exclude carcinoma [16].

\subsection{Endometrial carcinoma}

Endometrial carcinoma is the most common gynecological cancer in the United States. This represents only $1.5 \%$ of cancer deaths owing to its early detection. Endometrial carcinoma usually occurs in postmenopausal women with an average age of onset in the sixth decade of life. Sonohysterography represents an effective tool for the evaluation for endometrial carcinoma in the setting of postmenopausal bleeding [17]. Endometrial carcinoma is usually visualized as a diffuse heterogeneous lesion. However, a more focal form can also be seen. Focal forms are usually broad-based but can also be polypoid (Fig. 5). These lesions often distort the endometrial-myometrial interface. It must be noted that there is no absolute diagnostic appearance of carcinoma. Sometimes abnormal appearing masses may not be carcinoma and small foci of carcinoma may be present in lesions that look like simple polyps. Biopsy is always needed to establish the diagnosis of carcinoma.

\subsection{Adhesions}

Adhesions usually appear as mobile, thin, echogenic bands crossing the endometrial cavity (Fig. 6). Thick, broad-based bridging bands are a less commonly encountered pattern. It is difficult to distend the endometrial cavity when the severity of adhesions progresses [6]. Adhesions can be associated with endometrial scars, which have a variable appearance, ranging from small echogenic areas of focal endometrial thickening to regions of denuded endometrium.

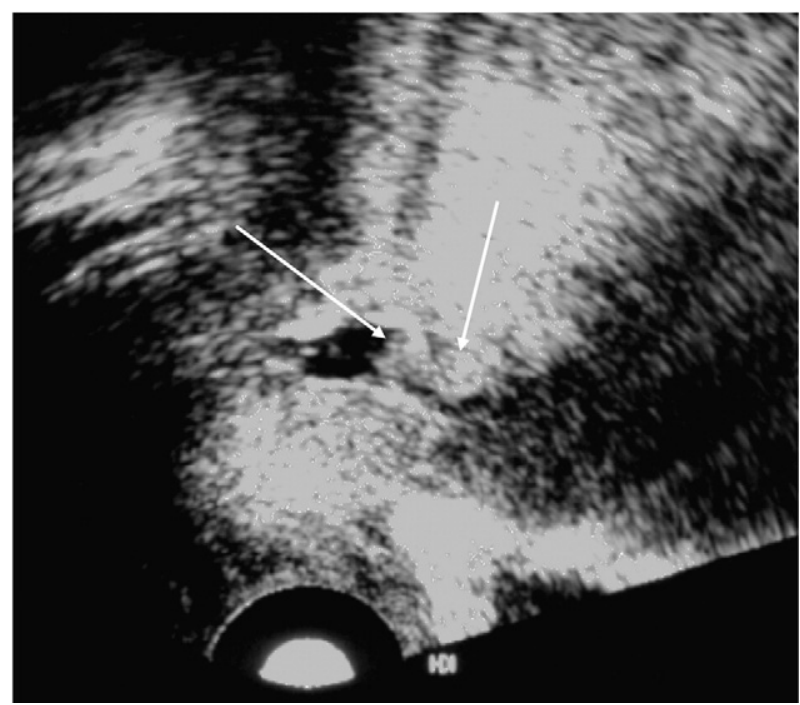

Fig. 5. 63-year-old female with postmenopausal bleeding. Sonohysterogram shows irregular focal thickening of anterior endometrium (arrows), representing endometrial carcinoma.

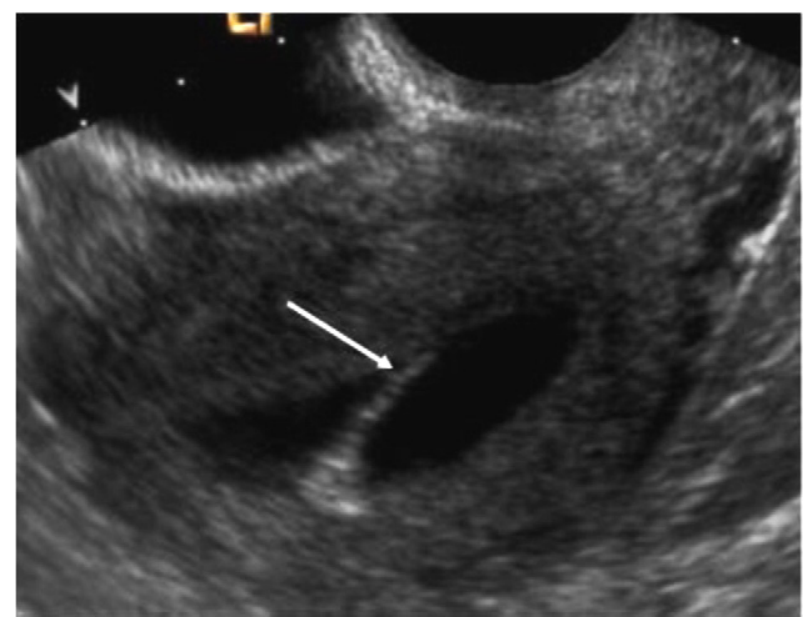

Fig. 6. 32-year-old female with history of 2 abortions. Sonohysterogram shows a thin echogenic band seen extending from the posterior myometrium to the anterior myometrium, representing intrauterine synechiae.

\subsection{Endometrial osseous metaplasia}

Endometrial ossification is a rare pathological entity, which is most commonly associated with a recent history of abortion, either spontaneous or therapeutic. Chronic endometritis is another known etiology. Bone formation has also been observed in malignant müllerian tumor and teratoma. By sonohysterography, osseous metaplasia of the endometrium is often seen as a diffuse echogenic involvement of the endometrium with shadowing.

\subsection{Adenomyosis}

Adenomyosis is a common nonneoplastic gynecologic disease characterized by the migration of endometrial glands from the basal layer of the endometrium into the myometrium, associated with smooth muscle hyperplasia. The ectopic glands are located at least $2-3 \mathrm{~mm}$ below the endometrial-myometrial junction; the associated smooth muscle hyperplasia may produce asymmetric thickening of the uterus. Adenomyosis typically appears as a diffuse disease process resulting in a globular uterine enlargement. However, focal forms can also be encountered and mistaken for a leiomyoma. Adenomyosis is more common in parous women. Abnormal uterine bleeding and dysmenorrhea are the most common symptoms of adenomyosis. Adenomyosis often appears as small cystic areas in the subendometrial region of the myometrium, but is not well diagnosed sonographically (Fig. 7).

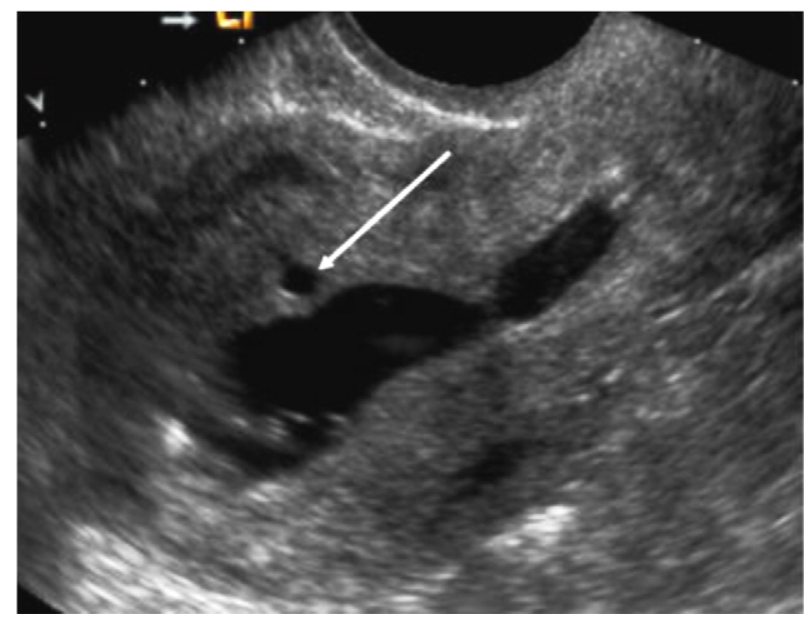

Fig. 7. 33-year-old female with abnormal uterine bleeding. Sonohysterogram shows anechoic area in the anterior subendometrial region, consistent with adenomyosis. 


\subsection{False positive findings}

False positive findings (i.e., suspected pathology not confirmed by hysteroscopy) have been attributed to blood clots, intrauterine debris, mucus plugs, shearing of normal endometrium, thickened endometrial folds, and misidentified endometrial fragments [18]. If clots or mucous are suspected, real-time imaging of catheter movement within the uterine cavity may demonstrate dislodgement of those foci, and allow their distinction from polyps or other abnormalities.

\section{Conclusion}

Saline infusion sonohysterography is playing an important role in the clinical evaluation of women presenting with abnormal vaginal bleeding or incidental findings of intracavitary lesions found on a routine pelvic ultrasound.

Without this technique, ultrasound provides only limited evaluation of the endometrium. Using representative examples, the utility, clinical usefulness, and technical pitfalls of sonohysterography have been described.

\section{References}

[1] Parsons AK, Lense JJ. Sonohysterography for endometrial abnormalities: preliminary results. J Clin Ultrasound 1993;21(2):87-95.

[2] Syrop CH, Sahakian V. Transvaginal sonographic detection of endometrial polyps with fluid contrast augmentation. Obstet Gynecol 1992;79(6):1041-3.

[3] Goldstein RB, Bree RL, Benson CB, Benacerraf BR, Bloss JD, Carlos R, et al. Evaluation of the woman with postmenopausal bleeding. JUltrasound Med 2001:20(10):1025-36.

[4] Goldstein SR, Zeltser I, Horan CK, Snyder JR, Schwartz LB. Ultrasonography-based triage for perimenopausal patients with abnormal uterine bleeding. Am J Obstet Gynecol 1997;177(1):102-7.
[5] Erdem M, Bilgin U, Bozkurt N, Erdem A. Comparison of transvaginal ultrasonography and saline infusion sonohysterography in evaluating the endometrial cavity in pre- and postmenopausal women with abnormal uterine bleeding. Menopause 2007; 14(5):846-52.

[6] Salle B, Gaucherand P, de Saint Hilaire P, Rudigoz RC. Transvaginal sonohysterographic evaluation of intrauterine adhesions. J Clin Ultrasound 1999;27(3):131-4.

[7] Darwish AM, Youssef AA. Screening sonohysterography in infertility. Gynecol Obstet Invest 1999;48(1):43-7.

[8] Alborzi S, Dehbashi S, Parsanezhad ME. Differential diagnosis of septate and bicornuate uterus by sonohysterography eliminates the need for laparoscopy. Fertil Steril 2002;78(1):176-8.

[9] Alatas C, Aksoy E, Akarsu C, Yakin K, Alsoy S, Hayran M. Evaluation of intrauterine abnormalities. Hum Reprod 1997;12(3):487-90.

[10] Dessole S, Farina M, Rubattu G, Cosmi E, Ambrosini G, Nardelli GB. Side effects and complications of sonohysterosalpingography. Fertil Steril 2003;80(3):620-4.

[11] Gronlund L, Hertz J, Helm P, Colov N. Transvaginal sonohysterography and hysteroscopy in the evaluation of female infertility, habitual abortion, or metrorrhagia: a comparative study. Acta Obstet Gynecol Scand 1999;78(5):415-8.

[12] Davis PC, O'Neill MJ, Yoder IC, Lee SI, Mueller PR. Sonohysterographic Findings of Endometrial and Subendometrial Conditions. Radiographics 2002;22(4):803-16.

[13] Sohaey R, Woodward P. Sonohysterography: technique, endometrial findings, and clinical applications. Semin Ultrasound CT MR 1999;20(4):250-8.

[14] Lev-Toaff AS, Toaff ME, Liu JB, Merton DA, Goldberg BB. Value of sonohysterography in the diagnosis and management of abnormal uterine bleeding. Radiology 1995;201(1):179-84.

[15] O'Connoll LP, Fries MH, Zeringue E, Brehm W. Triage of abnormal postmenopausal bleeding: a comparison of endometrial biopsy and transvaginal sonohysterography versus fractional curettage with hysteroscopy. Am J Obstet Gynecol 1998;178(5): 956-61.

[16] Maksem JA, Meiers I, Robboy SJ. A primer of endometrial cytology with histological correlation. Diagn Cytopathol 2007;35(12):817-44.

[17] Berry E, Lindheim SR, Connor JP, Hartenbach EM, Schink JC, Harter J, et al. Sonohysterography and endometrial cancer: incidence and functional viability of disseminated malignant cells. Am J Obstet Gynecol 2008;199(3) :240.e1-8.

[18] Lindheim SR, Morales AJ. Comparison of sonohysterography and hysteroscopy: lessons learned and avoiding pitfalls. J Am Assoc Gynecol Laparosc 2002;9(2):223-31. 